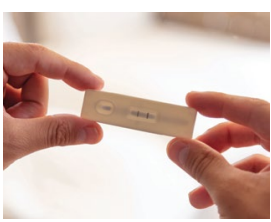
CRISPR tests COVID on the go p1031

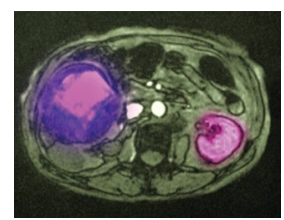

First von HippelLindau drug p1032

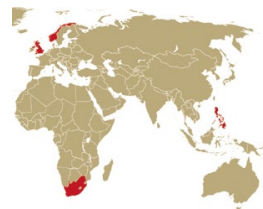

Biotech news from around the world p1035

\title{
Startups set off new wave of mRNA therapeutics
}

\author{
After the vaccine triumphs of Pfizer/BioNTech and Moderna, a raft of startups is developing mRNA, circular RNA \\ and self-amplifying RNA therapeutics.
}

E mboldened by the success of mRNA vaccines for COVID-19, the French drug giant Sanofi announced plans last month to pay $\$ 3.2$ billion for Translate Bio, an mRNA medicines specialty firm. The move comes hot on the heels of Sanofi's launch of a new $\$ 475$ million-per-year mRNA Center of Excellence for vaccines and the acquisition of Tidal Therapeutics, a three-year-old mRNA-focused spinout from the Fred Hutchinson Cancer Research Center. Stiff competition could come not necessarily from COVID mRNA vaccine heavyweights, but from a cadre of new startups, all of which are building next-generation RNA platform technologies designed to solve some of the unique challenges associated with using conventional mRNA tools as therapeutics.

For more than a decade, Moderna and many industry insiders have touted the promise of mRNA as a drug that could transform the body's cells into protein-production factories. The lightning-quick development of COVID-19 vaccines by Moderna and Pfizer/BioNTech demonstrated the power of this approach in infectious disease-but as a preventative measure, not to treat established disease. As yet, no mRNA drug candidate designed to replace or supplement a deficient disease-linked protein has advanced past first-in-human testing, nor has any mRNA-based product that encodes a non-native therapeutic protein, such as a monoclonal or bispecific antibody or a Cas endonuclease for gene editing.

Now, thanks to a deeper understanding of RNA and inventive ways of engineering the molecule, using mRNA as a therapeutic looks more attainable than ever, says Michael Ehlers, CSO of ATP (also known as Apple Tree Partners). But "it's relatively early days," he says, "and there's a lot of room for innovation."

Seeking to fill that innovation gap, Replicate Bioscience launched this month

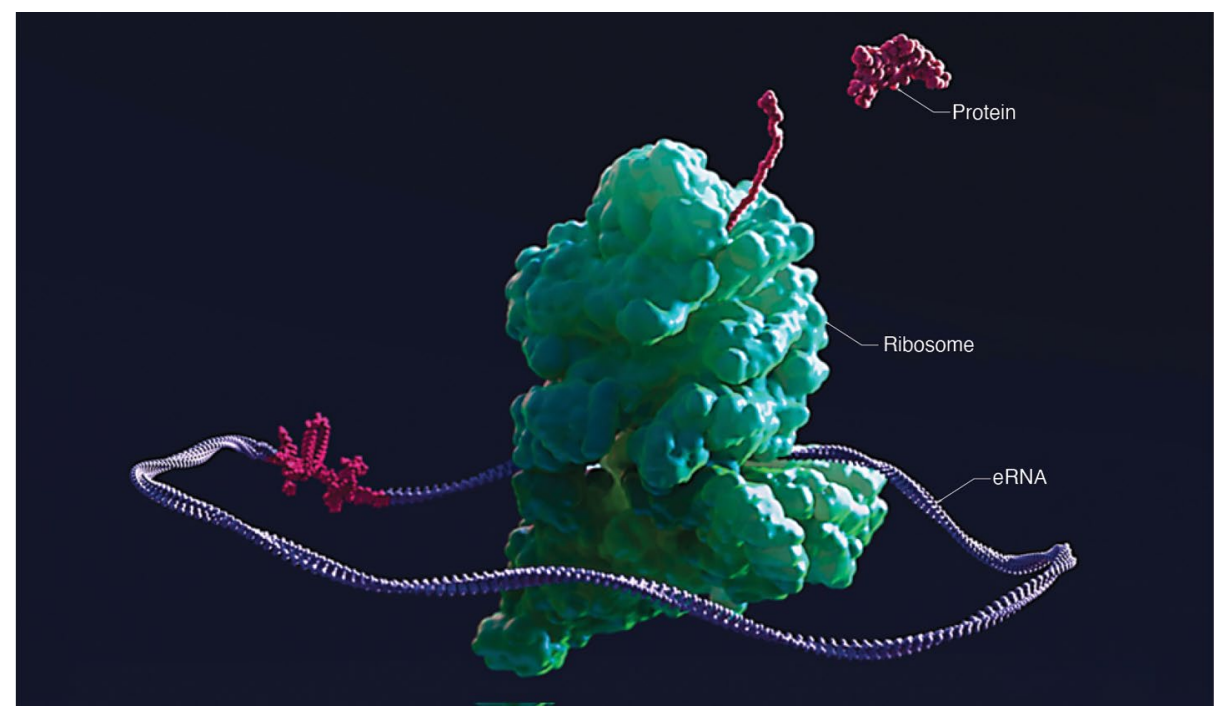

A ribosome translates 'endless' circular RNA (eRNA) into therapeutic proteins inside the body. Credit: Laronde

with plans to develop self-amplifying RNA (saRNA) constructs for preventing drug resistance in various cancers and for taming autoimmune disorders. The company, which debuted with $\$ 40$ million funding from ATP, joins the synthetic biology startups Strand Therapeutics and Kernal Biologics, plus a growing list of other fledgling firms centered around circular RNAs. These include Orna Therapeutics, unveiled in February with over \$100 million; Laronde, which launched in May with $\$ 50$ million; and Circ Bio, which remains in stealth mode.

Some of the distinct challenges of making mRNA therapeutics compared with mRNA vaccines include stabilizing the molecules for lasting therapeutic effects and maximizing potency to minimize inflammatory reaction at the injection site while still achieving high levels of protein expression. As for the encoded protein itself, it must be functional with the relevant post-translational modifications, splicing and expression in the right cellular location, avoiding off-target expression to preclude side effects.

But it is the short-lived nature of the molecule, experts say, that is the main problem with conventional mRNA. Intrinsically unstable, mRNA is prone to enzymatic degradation inside the body, which means that most therapeutics built around the technology typically only stick around inside cells for a few days. That might be enough time for some applications - with prophylactic vaccines, for instance, only a small burst of antigen production is sufficient to trigger lasting immune responses-but not for chronic disease treatment, where persistent protein expression is required. Repeated dosing could theoretically get around the issue of transient activity, but the toxicities associated with mRNA and their associated lipid nanoparticle delivery systems typically preclude those kinds of frequent administration strategies. 


\section{Box 1 | Self-amplifying RNA vaccines}

A few self-amplifying RNA vaccines have entered human trials, mostly for COVID-19 and nearly all built around a single alphavirus expression system derived from the Venezuelan equine encephalitis virus. Compared with conventional mRNA vaccines, the expectation is that the lower dosages theoretically needed for self-amplifying RNA vaccines may have advantages in terms of reactogenicity at the injection site and higher numbers of vaccine doses that can be manufactured for distribution. At least one of these COVID-19 candidates, from VaxEquity, was abandoned after it failed to elicit a sufficient immune response-and VaxEquity co-founder Anna Blakney thinks the expression vector might be to blame. "I think it just needs a lot more optimization," says Blakney, an RNA bioengineer at the University of British Columbia in Vancouver, Canada. Some companies are now refining the molecular design of saRNA constructs in an effort to do just that.

One decades-old idea for extending the duration of mRNA expression (while also improving tolerability) has been to deliver low doses of saRNA. Derived from alphavirus expression vectors, these constructs contain RNA templates that encode therapeutic proteins, just like conventional mRNAs do. But they also contain replicase genes that allow the RNAs to make copies of themselves in the cell cytoplasm. Most of this work to date has focused on vaccines (Box 1). But in principle, the same strategy should work for personalized cancer therapeutics, such as neoantigen vaccines, as well.

At one therapeutics-oriented startup, Replicate Bioscience, CEO Nathaniel Wang and his colleagues have been reconsidering the alphavirus expression vectors that power saRNA technologies. Their effort involved a systematic screen of more than 15 different kinds of alphaviruses, each encoding a range of different transgenes. They found that different viruses worked best for different protein payloads and different therapeutic applications.

According to Wang, a "completely new" type of alphavirus, as yet undisclosed, underpins the company's lead product line, a series of cancer vaccines designed to prevent drug resistance for patients receiving immunotherapies or targeted anti-cancer agents. On top of the alphavirus backbone, Replicate scientists have incorporated
BioNTech, for example, in collaboration with partners at TRON, a non-profit research organization in Mainz, Germany, has developed a trans-amplifying strategy that involves two RNA vectors: one self-amplifying with the replicase machinery of an alphavirus, the other a more conventional, non-replicating mRNA. The two genes still work together, but splitting the design into two distinct RNAs creates a huge manufacturing advantage, explains BioNTech CEO Uğur Şahin. That's because very long RNA transcripts, such as self-amplifying RNAs tailor-made to address, say, a new strain of influenza, can be difficult to produce at scale in a speedy fashion, unlike shorter mRNAs. With the trans-amplifying approach, Şahin says, only one kind of replicase-encoding RNA is ever required. Thus, companies can "keep it in stock." Then, for each flu season, or in the event of a new zoonotic outbreak, only tiny amounts of bespoke mRNA have to be made anew.

various tumor type-specific transgenes, each encoding up to a dozen different mutated proteins that typically drive resistance in different cancer contexts.

The company is also working on biotherapeutics for oncology and immunological conditions. And thanks to improvements in vector design and delivery systems, Wang anticipates achieving "orders of magnitude jumps" in expression levelsand thus potency-as compared with what is possible with conventional saRNA designs in clinical testing today.

Strand, for its part, is designing genetic circuits and small-molecule chemical inducers to enable more precise control of the location, timing, intensity and duration of the platform's therapeutic activities. Part of that engineering involves modulating the sequence and structural elements of the saRNA vector itself, as Strand has done for its lead product, an intratumoral therapy designed to spur an inflammatory response in the cancer microenvironment. (Under the terms of a deal cemented earlier this year, BeiGene has development and commercial rights to the immunostimulatory therapeutic in most Asia-Pacific markets). But several other research-stage assets also include genetically programmed logic circuits that can sense microRNA signatures to trigger cell type-specific expression of encoded proteins, explains co-founder and CEO Jake Becraft.
What's more, says Becraft, the company is "platform agnostic." Although Strand's first products involve saRNA, "the technology itself can be applied across all different types of messenger RNAs," he says. Strand announced its series A financing of $\$ 52$ million in June.

Like Strand, Kernal Biologics - which raised \$10.5 million in seed funding last year-is developing new kinds of mRNA therapeutics for cancer. But in Kernal's case, this involves engineering mRNA in two distinct ways. First, the company eliminates any sequence motifs that might trigger recognition by Toll-like receptors (TLRs) found in human cells, creating what CEO Yusuf Erkul describes as "stealth mRNA." Then Kernal tailors mRNA molecule to take advantage of a quirk of ribosome biology in p53-deficient tumor tissues.

To do this, the company seized on epigenetic alterations of ribosomes in cancer cells lacking a functioning version of p53 that mean they read through 'stop' codons that would normally terminate the translation process. By designing mRNA open reading frames downstream of such stop codons, Erkul says that his company creates therapeutics that become preferentially active in cancerous tissues-and he has unpublished data from experiments conducted with leukemic and normal cells aboard the International Space Station to prove the constructs remain cancer cell specific, even in the stress environment of microgravity, which is known to alter ribosome biogenesis. Erkul plans to present those results at the 9th International mRNA Health Conference in November.

Rather than trying to improve on traditional mRNA designs, several companies have opted instead to pursue a different therapeutic construct: circular RNA. These contiguous rings of single-stranded RNA lack the caps and polyadenosine tails that RNA-degrading enzymes usually latch onto. And "because it doesn't have ends," says Daniel Anderson, a bioengineer at the Massachusetts Institute of Technology in Cambridge and a scientific cofounder of Orna, "it doesn't get chewed up" as quickly, leading to more robust and durable protein expression. Unlike linear mRNAs, which ribosomes scanning from the $5^{\prime}$ end, circular RNAs must recruit the translation machinery with internal ribosome entry sequences to facilitate protein production.

At Laronde, mouse experiments conducted with the company's 'endless RNA' - as the firm has branded its circular RNA constructs-have produced "persistent protein expression in vivo for many weeks 
and months after a single administration," according to CEO Diego Miralles. "We believe this could have a huge impact in how we think about protein therapeutics, opening a whole world of possibilities in how we treat diseases and relieve human suffering," he says.

The idea of using engineered circular RNAs as potential therapeutic agents dates back more than 25 years, but the strategy never gained much traction back then. Virologist Peter Sarnow, for example, filed for some early intellectual property around the platform after showing in 1995 that circular RNA could prompt protein production in human cells, but his institution at the time, the University of Colorado in Denver, discontinued the patent because there was no commercial interest. "Bummer!" says Sarnow, now at the Stanford University School of Medicine.

More recently, as the limitations of linear mRNA have become apparent, entrepreneurs are taking a second look at RNA circles. "It really has caught people's fancy now in ways it didn't before," says Manny Ares, an RNA biologist at the University of California, Santa Cruz, who in the 1990s developed some of the first methods for synthesizing circular RNAs.

In addition to the prolonged expression dynamics of circular RNA, Orna CEO Tom Barnes points to several other benefits of the platform compared with linear mRNA. For starters, the manufacturing is more cost effective: "Because the RNAs autocatalytically circularize at high efficiency," he says, there is no need for further steps with expensive reagents. "There is no capping, there is no tailing" - referring to chemical modifications at the $5^{\prime}$ and $3^{\prime}$ ends of mRNA molecules.

Circular RNAs can also evade the innate immune responses with or without the addition of modified nucleotides, thus adding further cost savings. Plus, Barnes notes, the technology can accommodate long sequences - up to 5 or possibly even 10 kilobases, as Anderson and Alexander Wesselhoeft, Orna's cofounder and director of molecular biology, have shown-which could open up new therapeutic possibilities.

"To me, it's clearly better," says Robert Kruse, a physician-scientist at Harvard Medical School in Boston, of circular RNA. But even though Kruse holds patents around the technology, he remains circumspect about whether the platform offers much more than incremental advances over linear mRNA. "The half-life is a great advantage. But beyond frequency of dosing," he says, "I don't know whether it will truly unlock a new therapeutic application."
In the meantime, some companies are findings new therapeutic applications for mRNA simply by reimagining what is possible with existing technologies. At Intellia Therapeutics, for example, the company's in vivo gene-editing therapies are all based on mRNAs that encode the Cas9 endonuclease enzyme. (Others have traditionally used viral vectors or ribonucleoprotein complexes to deliver the CRISPR machinery instead.)

The company reported in June results from six patients with the rare and fatal condition transthyretin amyloidosis who received infusions of the genome-editing RNA construct. The data showed that the mRNAs were successfully transformed into active Cas9 proteins that cut DNA at a specific target sequence, the location determined by an engineered guide RNA that was co-formulated alongside the mRNA inside liver-targeted lipid nanoparticles. The therapy introduced frameshift mutations at the disease-associated gene in liver cells, leading to declines in circulating levels of the aberrant protein.

Notably, in this context, the fleeting nature of mRNA is desirable, not a drawback. "I just want to have a pulse of Cas9 protein being made," says Laura Sepp-Lorenzino, CSO of Intellia. Otherwise the enzyme could spark immune reactions or off-target effects. "For me," she says, "short duration of expression is exactly what I'm looking for."

The same goes for Geoffrey von Maltzahn, CEO of Tessera Therapeutics and a general partner at Flagship Pioneering. Tessera is using a protein borrowed from the retrotransposition machinery of mobile genetic elements to make targeted insertions of therapeutic sequences into the genome. In the company's preclinical-stage products, that protein too is encoded in mRNA. "We're in the year of nucleic acids," von Maltzahn says, "and there's a ton of synergy" between different platform technologies. "Brought into the realm of gene writing and the broader area of genomic medicine, it's our view that the future is definitively going to be powered by RNA therapeutics."

Sanofi and others are betting on that future as well.

As Ehlers points out, targeted delivery of mRNA to most organs and tissues remains a challenge, which "will limit applications and indications." But, he says, "we see lots of reasons for optimism." RNA-based drugs are "going to be a big therapeutic class-no question."

\section{Elie Dolgin}

Somerville, MA, USA

Published online: 2 September 2021

https://doi.org/10.1038/s41587-021-01056-6

\section{CRISPR-based portable COVID tests}

Two new CRISPR-based tests developed by academic groups have each taken a step toward providing portable, low-cost, sensitive SARS-CoV-2 diagnostics. A team at MIT and the Wyss Institute led by James J. Collins, publishing in Science Advances, describes an at-home saliva test that detects and reports the presence of different viral variants within an hour. The other COVID-19 diagnostic, published in Nature Chemical Biology by Jennifer Doudna's group at the University of California, Berkeley, is a point-of-care diagnostic that enables results using swab testing within $20 \mathrm{~min}$.

The MIT team developed the miSHERLOCK (short for minimally instrumented SHERLOCK) test. It is based on the CRISPR platform developed by Sherlock Biosciences (co-founded by Collins and Feng Zhang from the Broad Institute). The new assay uses Cas12a guide RNA to distinguish different variants with a limit of detection comparable to that of the gold-standard RT-PCR test: 1 molecule per microliter in unprocessed saliva. The team aimed to show it was possible to build a low-cost CRISPR-based test involving minimal instrumentation to detect SARS-CoV2 in raw samples, with results delivered to a smartphone app.

The competing paper from the Doudna lab combines two unrelated CRISPR nucleases-RNA-guided Cas13 and Csm9-in a tandem assay to provide a simpler alternative to PCR-based methods in a portable microfluidic chip. The one-step assay, named Fast Integrated Nuclease Detection in Tandem, differs from other approved CRISPR-based COVID assays in that it does not require an amplification step. The tandem nuclease technology boosts detection and amplifies the signal such that it can detect 30 copies per microliter of target RNA, well below the threshold of 100 copies per microliter necessary for diagnostic surveillance.

Published online: 9 September 2021 https://doi.org/10.1038/s41587-021-01061-9 